\title{
POLIANTHES OAXACANA Y P. GEMINIFLORA VAR. PUEBLENSIS (AGAVACEAE), TAXA NUEVOS DE MÉXICO
}

\author{
Abisaí J. García-Mendoza ${ }^{1}$ y Eloy Solano ${ }^{2}$ \\ ${ }^{1}$ Universidad Nacional Autónoma de México, Instituto de Biología, Jardín Botánico \\ Apdo. postal 70-614, Delegación Coyoacán, 04510 México, D.F. \\ abisai@ibiologia.unam.mx \\ ${ }^{2}$ Universidad Nacional Autónoma de México, Facultad de Estudios Superiores \\ Zaragoza, Herbario FEZA, Carrera de Biología \\ Apdo. postal 9-020, Delegación Iztapalapa, 09230 México, D.F. \\ solanoec@correo.unam.mx
}

\section{RESUMEN}

Se describen e ilustran dos taxa nuevos para la ciencia: Polianthes oaxacana y $P$. geminiflora var. pueblensis. Polianthes oaxacana, del estado de Oaxaca, se ubica en el subgénero Bravoa; se caracteriza por sus flores tubulares, bilateralmente simétricas, con la parte distal campanulada y anteras adpresas a la porción superior del limbo del perigonio. Se compara con P. bicolor Solano \& García-Mend. y P. mexicana Zucc.

Polianthes geminiflora var. pueblensis del estado de Puebla, pertenece al subgénero Bravoa; se distingue de las otras variedades de P. geminiflora por sus hojas lineares con el margen papiloso a regularmente denticulado y por algunas características anatómicas.

Palabras clave: Agavaceae, Bravoa, México, Oaxaca, Polianthes, Puebla.

\section{ABSTRACT}

Two new taxa, Polianthes oaxacana and $P$. geminiflora var. pueblensis, are described and illustrated. Polianthes oaxacana, from the state of Oaxaca, belongs to subgenus Bravoa and is characterized by its tubular, bilaterally symmetrical flowers, the distal part campanulate, and anthers appressed to the upper side of the perigonium limb. The new species is compared with Polianthes bicolor Solano \& García-Mend., and P. mexicana Zucc. 
Polianthes geminiflora var. pueblensis belongs to subgenus Bravoa and is distinguished from other varieties of the species by its linear leaves with papillose or regurlarly denticulate margin and some anatomical characters.

Key words: Agavaceae, Bravoa, Mexico, Oaxaca, Polianthes, Puebla.

En el proceso de revisión taxonómica del género Polianthes L., se recolectaron y estudiaron especímenes provenientes de los estados de Oaxaca y Puebla, que resultaron pertenecer a una especie y una variedad nuevas que se describen a continuación:

Polianthes oaxacana García-Mend. \& E. Solano sp. nov. Fig. 1.

Planta herbacea perennis, rosulata. Folia 3-4 per rosulam, (40-)60-70 cm longa, 1-1.5(-2) cm lata, linearia, semisucculenta. Flores tubulares, bilateraliter symmetrici, adpressi vel diffusi, (4.5-)5-5.2 cm longi, ad basem loborum 1-1.4 $\mathrm{cm}$ lati, extus rosei, intus lutei, tubo (3-)3.8-4.2 cm longo, ad basem ascendenti, prope dimidium arcuato, distaliter campanulato, lobis 5-7 $\mathrm{mm}$ longis, 4-6 mm latis, ovatis vel orbiculatis, subaequalibus, patentibus, stamina inclusa, filamentis 1-1.2 $\mathrm{cm}$ longis, prope faucem sitibus variis tubi insertis, inferiore (1.5-)2-2.1 cm supra apicem ovarii, superiore (2-)2.5-2.7 cm supra apicem ovarii, antheris sub anthesi in parte distali limbi perigonii adpressis.

Planta herbácea, perenne. Cormo (1.5-)4-5 cm de largo, 1-2 cm de diámetro, cilíndrico, con yemas de crecimiento y raíces engrosadas de $8-12 \mathrm{~cm}$ de largo; bulbo 5-8 cm de largo, 2-3 cm de diámetro, ovoide, cubierto por las bases de las hojas secas. Hojas 3-4 por roseta, (40-)60-70 cm de largo, 1-1.5(-2) cm de ancho, lineares, semisuculentas, arqueadas, verdes con la base blanquecina; margen papiloso al microscopio, hialino, en ocasiones rojizo; ápice atenuado, agudo, con un engrosamiento suave. Inflorescencia espigada, 140-180 cm de largo, 3-7 nudos florales, pedúnculo $120-150 \mathrm{~cm}$, con 6-7 brácteas, sucesivamente más cortas hacia el ápice, similares a las hojas, la basal (30-)45-60 cm de largo, 0.5-0.7 cm de ancho, linear, margen papiloso al microscopio, ápice con un engrosamiento suave; porción fértil 10-30 cm, con 6-14 flores, brácteas florales 0.5-1.5 cm de largo, 2-3 mm de ancho, apiculadas. Flores 4.5-5.2 cm de largo, 1-1.4 cm de ancho en la base de los lóbulos, geminadas, sésiles, tubulares, bilateralmente simétricas, tubo del perigonio 


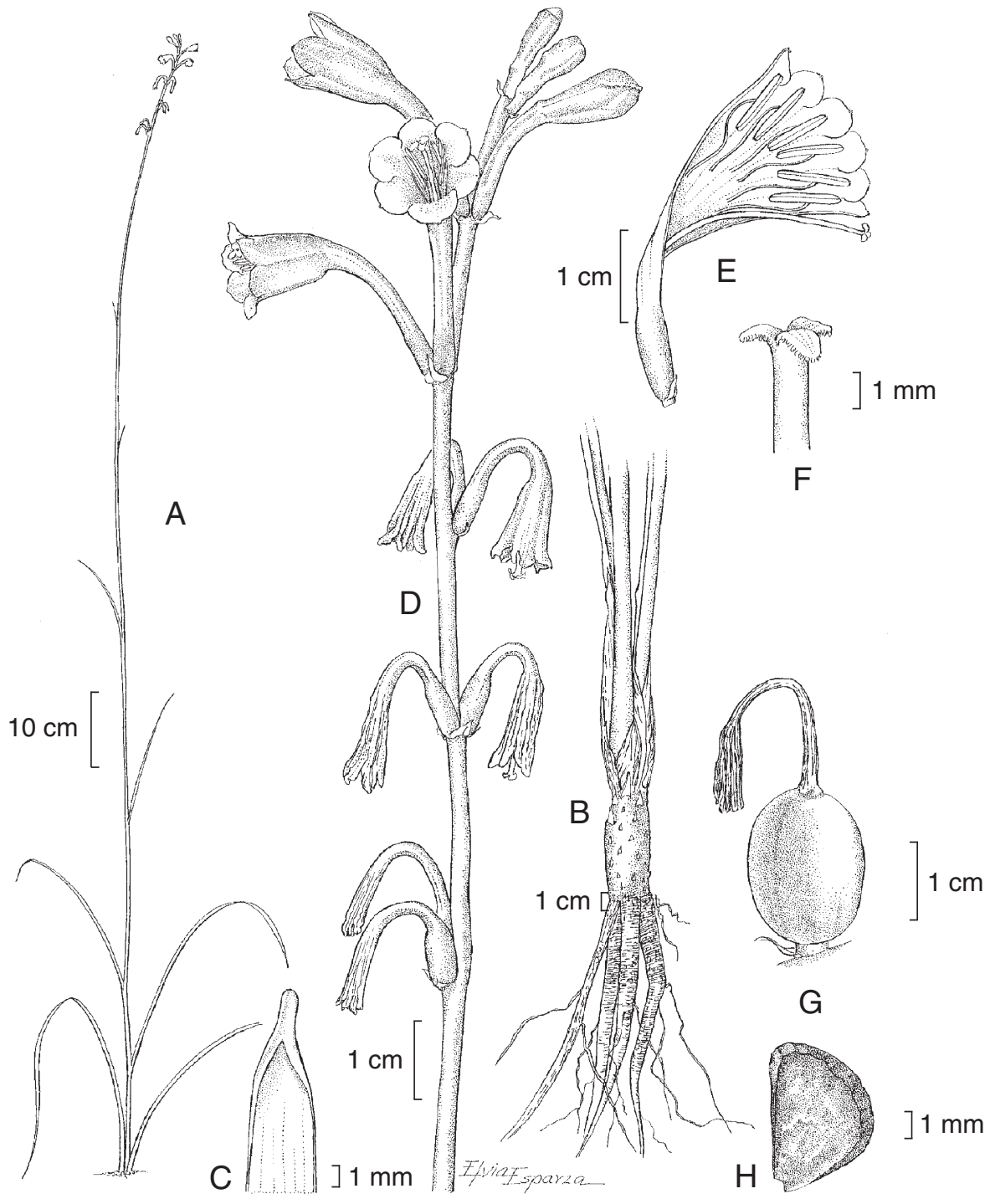

Fig. 1. Polianthes oaxacana. A. hábito; B. cormo y bulbo con raíces engrosadas; C. ápice de la hoja; D. inflorescencia; E. flor disecada. F. estigma trilobado; G. fruto; H. semilla. Ilustración basada en los ejemplares A. García-Mendoza y E. Solano 6970 (MEXU) y E. Solano y A. García-Mendoza 1255 (FEZA, MEXU). 
en la base ascendente, cerca de la mitad arqueado, con la porción distal campanulada, de color rosado en el exterior, amarillento en el interior, con tintes violáceos cuando seco, sin olor; ovario (6-)9-13 mm de largo, 4-6 mm de diámetro, prolongándose ligeramente en el tubo; tubo (3-)3.8-4.2 cm de largo, ampliamente dilatado en la porción distal, 1-1.4 cm de ancho en la base de los lóbulos, lóbulos 5-7 mm de largo, 4-6 mm de ancho, ovados a orbiculares, subiguales, mucho más cortos que el tubo, extendidos, con el ápice cuculado, agudo o apiculado, ligeramente engrosado, papiloso; estambres inclusos, porción libre de los filamentos 1-1.2 cm de largo, insertos en el tubo a diferentes niveles cerca de la garganta, el inferior (1.5-)2-2.1 cm por arriba del ápice del ovario y el superior a (2-)2.5-2.7 cm, rosados; anteras (7-)9$10 \mathrm{~mm}$ de largo, $1 \mathrm{~mm}$ de ancho, lineares, verde-amarillentas, en antesis adpresas a la porción superior del limbo del perigonio; estilo 3.5-4.2 cm de largo, filiforme, blanco-amarillento, incluso, en flores senescentes sobrepasando a los lóbulos hasta por $1.2 \mathrm{~cm}$; estigma trilobado, lóbulos $1.5-2 \mathrm{~mm}$ de largo, $1 \mathrm{~mm}$ de ancho, oblongos, aplanados, recurvados, margen papiloso. Frutos capsulares, 1.5-2 cm de largo, 1.3$1.6 \mathrm{~cm}$ de diámetro, oblongos a globosos, inmaduros verde-rojizos, coronados por el perigonio persistente. Semillas 4-6 mm de largo, 3.5-4 $\mathrm{mm}$ de ancho, deltoides o semicirculares, planas, negras.

Tipo: México, Oaxaca: distrito de Zimatlán, municipio de San Pedro el Alto, 7 $\mathrm{km}$ al NE de San Vicente Lachixío, 2390 m, bosque de Pinus-Quercus, 16 septiembre 2000, A. García-Mendoza y E. Solano 6970 (holotipo: MEXU).

Paratipos: México, Oaxaca: distrito de Sola de Vega, $4 \mathrm{~km}$ al SO de San Vicente Lachixío, camino a Tlacuache, $R$. Cedillo y R. Torres 1784 (MEXU); distrito de Zimatlán, municipio de San Pedro el Alto, $8 \mathrm{~km}$ al NE de La Cofradía, camino a San Pedro el Alto, A. García-Mendoza y E. Solano 7295 (MEXU); misma localidad, A. García-Mendoza 7408 (MEXU); distrito de Zimatlán, comunidad de San Pedro el Alto, Cañada El Frijolón, $8 \mathrm{~km}$ al NO de La Cofradía, A. Miranda y $O$. Hernández M. 245 (CHAP); distrito de Zimatlán, comunidad de San Pedro el Alto, Pueblo Viejo, $6 \mathrm{~km}$ al N de La Cofradía, camino La Cofradía-San Pedro, L. Salazar M. 18 (CHAP, MEXU); distrito de Zimatlán, municipio de San Antonino el Alto, 17 $\mathrm{km}$ al NO de El Trapiche o $13 \mathrm{~km}$ al SE de San Antonino, E. Solano y A. GarcíaMendoza 1255, 1255A (FEZA, MEXU, OAX).

Fenología. Florece de septiembre a octubre y fructifica de noviembre a diciembre. 
Hábitat y distribución. Polianthes oaxacana crece en las laderas de las montañas, sobre suelos pardo-rojizos de textura arenosa o limoso-arenosa, dentro del bosque de Pinus-Quercus con Alnus, Arbutus, Cornus, Dalea y Pernettya, en altitudes de 2390 a $2600 \mathrm{~m}$. Aparentemente se restringe a las montañas de la Sierra Madre del Sur, en los distritos de Sola de Vega y Zimatlán, Oaxaca. Es la especie con distribución más meridional del género y también una de las que crecen a mayor altitud, superada sólo por Polianthes longiflora, que ha sido recolectada a $2700 \mathrm{~m}$ (Solano, 2000).

Polianthes se divide en dos subgéneros (Verhoek-W., 1975; Solano, 2000). El subgénero Polianthes se caracteriza por su inflorescencia en espiga, estambres insertos por debajo de la base de los lóbulos y flores blancas o amarillas en antesis, generalmente fragantes. El subgénero Bravoa se distingue por su inflorescencia en racimo, estambres insertos en la base o porción media del tubo y flores anaranjadas, anaranjado-verdosas con el ápice verde, amarillentas o rosadas, generalmente sin fragancia. Polianthes oaxacana se reconoce por sus flores sésiles, bilateralmente simétricas, adpresas a difusas, tubo en la base ascendente, arqueado cerca de la mitad, con la porción distal campanulada, de color rosado en el exterior, amarillento en el interior. Estas características son intermedias entre los dos subgéneros, sin embargo, decidimos incluirla dentro de Bravoa por la inserción de los estambres, el color de las flores y la ausencia de aroma.

Morfológicamente Polianthes oaxacana se relaciona con Polianthes bicolor Solano \& García-Mend., de la que se distingue por las características mostradas en el Cuadro 1. Asimismo, muestra similitudes con Prochnyanthes mexicana (Zucc.) Rose (Polianthes mexicana Zucc.), como son: la simetría bilateral de las flores en antesis, el tubo abruptamente expandido en la parte distal y estambres agrupados en la porción superior del limbo del perigonio. Prochnyanthes con una sola especie, se diferencia de Manfreda por la presencia de flores geminadas, pediceladas y estambres inclusos (Verhoek-W., 1975; McVaugh, 1989b), y de Polianthes por las flores campanuladas de color verdoso o púrpura. La especie aquí descrita posee características morfológicas intermedias entre Polianthes y Prochnyanthes, por lo tanto, se apoya la propuesta de reconocer un solo género: Polianthes. Los estudios fenéticos y cladísticos de Solano (en proceso) y moleculares de Bogler y Simpson (1996) y Eguiarte et al. (2000), concuerdan con la idea de que los géneros Polianthes y Prochnyanthes son grupos hermanos, por lo que taxonómicamente deben ser considerados como un solo género. 
Cuadro 1. Características comparativas de Polianthes oaxacana García-Mend. \& E. Solano, P. bicolor Solano \& García-Mend. y P. mexicana Zucc. Los datos de Polianthes bicolor están tomados de Solano y García-Mendoza (1998) y los de Polianthes (Prochnyanthes) mexicana en parte de McVaugh (1989b).

\begin{tabular}{|c|c|c|c|}
\hline Carácter & Polianthes oaxacana & Polianthes bicolor & Polianthes mexicana \\
\hline Número de hojas & $3-4$ & $3-6(-12)$ & $(1-) 2(-5)$ \\
\hline Hojas & $\begin{array}{l}(40-) 60-70 \times 1-1.5 \\
(-2) \mathrm{cm}, \text { lineares }\end{array}$ & $\begin{array}{l}(5-) 8-15 \times 0.6-1.4 \mathrm{~cm}, \\
\text { lanceoladas }\end{array}$ & $\begin{array}{l}20-50(-60) \times 1- \\
5(-7) \mathrm{cm}, \text { lineares, } \\
\text { lanceoladas } \\
\text { o elípticas, } \\
\text { subpecioladas }\end{array}$ \\
\hline $\begin{array}{l}\text { Tipo y largo de la } \\
\text { inflorescencia }\end{array}$ & espiga, $140-180 \mathrm{~cm}$ & $\begin{array}{l}\text { racimo, 20-40(-54) } \\
\mathrm{cm}\end{array}$ & racimo, (120-)200 cm \\
\hline Pedicelo de la flor & ausente & presente, $0.6-1.3 \mathrm{~cm}$ & $\begin{array}{l}\text { presente, } 0.3-4.5 \\
(-7) \mathrm{cm}\end{array}$ \\
\hline Flor & $\begin{array}{l}4.5-5.2 \mathrm{~cm}, \text { tubular, } \\
\text { con la porción distal } \\
\text { campanulada }\end{array}$ & $\begin{array}{l}\text { 2-2.6 cm, tubular, } \\
\text { con la porción distal } \\
\text { ensanchada }\end{array}$ & $\begin{array}{l}(2.5-) 3-4 \mathrm{~cm} \text {, tubular, } \\
\text { con la porción distal } \\
\text { campanulada }\end{array}$ \\
\hline Tubo del perigonio & $\begin{array}{l}(3-) 3.8-4.2 \mathrm{~cm} \text { largo } \\
\text { x } 1-1.4 \mathrm{~cm} \text { en la base } \\
\text { de los lóbulos }\end{array}$ & $\begin{array}{l}2-2.6 \mathrm{~cm} \text { largo x } 0.2- \\
0.4 \mathrm{~cm} \text { en la base de } \\
\text { los lóbulos }\end{array}$ & $\begin{array}{l}1.1-2.7 \text { x } 0.8-1.4 \\
(-1.8) \mathrm{cm} \text { en la base } \\
\text { de los lóbulos }\end{array}$ \\
\hline $\begin{array}{l}\text { Lóbulos del } \\
\text { perigonio }\end{array}$ & $\begin{array}{l}\text { 5-7 x 4-6 mm, } \\
\text { ovados a orbiculares }\end{array}$ & $\begin{array}{l}2-4 \times 2-4(6) \mathrm{mm} \text {, } \\
\text { orbiculares a } \\
\text { transversalmente } \\
\text { elípticos }\end{array}$ & $\begin{array}{l}3-10 \times 3-6 \mathrm{~mm} \text {, } \\
\text { deltoides }\end{array}$ \\
\hline $\begin{array}{l}\text { Inserción de } \\
\text { los filamentos } \\
\text { inferiores, por } \\
\text { arriba del ápice del } \\
\text { ovario }\end{array}$ & $(1.5-) 2-2.1 \mathrm{~cm}$ & $1.1-1.9 \mathrm{~cm}$ & $3-5(-7) \mathrm{mm}$ \\
\hline Fruto & $\begin{array}{l}1.5-2 \times 1.3-1.6 \mathrm{~cm}, \\
\text { oblongo a globoso }\end{array}$ & $1 \times 1 \mathrm{~cm}$, globoso & $\begin{array}{l}1-1.4 \text { x } 1-1.2 \mathrm{~cm}, \\
\text { globoso }\end{array}$ \\
\hline $\begin{array}{l}\text { Distribución } \\
\text { conocida }\end{array}$ & Oaxaca & Oaxaca & $\begin{array}{l}\text { Durango y Nayarit } \\
\text { a Michoacán y } \\
\text { Querétaro }\end{array}$ \\
\hline
\end{tabular}


En el estado de Oaxaca, además de Polianthes oaxacana, crece P. bicolor Solano \& García-Mend., que se distribuye en los distritos de Coixtlahuaca, Juxtlahuaca, Nochixtlán, Teposcolula y Tlaxiaco; probablemente también se encuentre $P$. geminiflora (Lex.) Rose var. geminiflora, especie que aún no ha sido recolectada en el estado, pero que se ha registrado en el municipio de Chila, estado de Puebla, a menos de $200 \mathrm{~m}$ del límite con el distrito de Huajuapan, Oaxaca ( $A$. García 7113 en MEXU). Las dos primeras especies son endémicas de Oaxaca, mientras que la tercera se distribuye de Durango a Guerrero, Veracruz, sur de Puebla y probablemente el noroeste de Oaxaca. Los tres taxa se pueden determinar con la siguiente clave.

1 Hojas (40-)60-70 cm de largo; tubo del perigonio (3-)3.8-4.2 cm de largo, 1-1.4 cm de ancho en la base de los lóbulos P. oaxacana

1 Hojas 5-30 cm de largo; tubo del perigonio $1.4-2.6 \mathrm{~cm}$ de largo, menor de $0.5 \mathrm{~cm}$ de ancho en la base de los lóbulos.

2 Hojas 4-6(-12) por roseta; tubo del perigonio 2-2.6 cm de largo, ensanchado por arriba de la base; lóbulos verdes; filamentos 1.8-2.5(-2.8) cm de largo, insertos a 1.1-1.9 $\mathrm{mm}$ por arriba del ápice del ovario P. bicolor

2 Hojas 2-4 por roseta; tubo del perigonio 1.4-2 cm de largo, tubular; lóbulos anaranjados; filamentos 0.5-1.5(-2.4) cm de largo, insertos a 2-5(-9) $\mathrm{mm}$ por arriba del ápice del ovario P. geminiflora var. geminiflora

Polianthes geminiflora (Lex.) Rose var. pueblensis E. Solano \& García-Mend. var. nov. Fig. 2.

Planta herbacea perennis, rosulata, 3-6 foliis per rosulam, 14-17 cm longis, 1-2 mm latis, linearibus, margine papillosis vel denticulatis. Flores $1.5-2.1 \mathrm{~cm}$ longi, ad basem loborum 1.5-3 mm lati. Fructus 1.2-1.7 cm longi, 0.9-1.1 cm lati. Semina 3-5 mm longa, 2-3 mm lata. Cellulae mesophylli tanninis carentes, basis pedunculi floralis cuticula laevi, rhaphidibus et tanninis carens.

Planta herbácea, perenne, $35-65 \mathrm{~cm}$ de alto. Cormo 1-3 cm de largo, 0.7-1 cm de diámetro, cilíndrico, con yemas de crecimiento y raíces engrosadas; bulbo 1-3 cm de largo, 1-2 cm de diámetro. Hojas 3-6 por roseta, 14-17 cm de largo, 0.1-0.2 cm de ancho, lineares, con algunas manchas purpúreas cerca de la base; margen papiloso a regularmente denticulado, envés papiloso; ápice agudo. Inflorescencia un racimo laxo, denso hacia su porción distal, 35-65 cm de largo, 2-5 nudos fértiles, el basal 


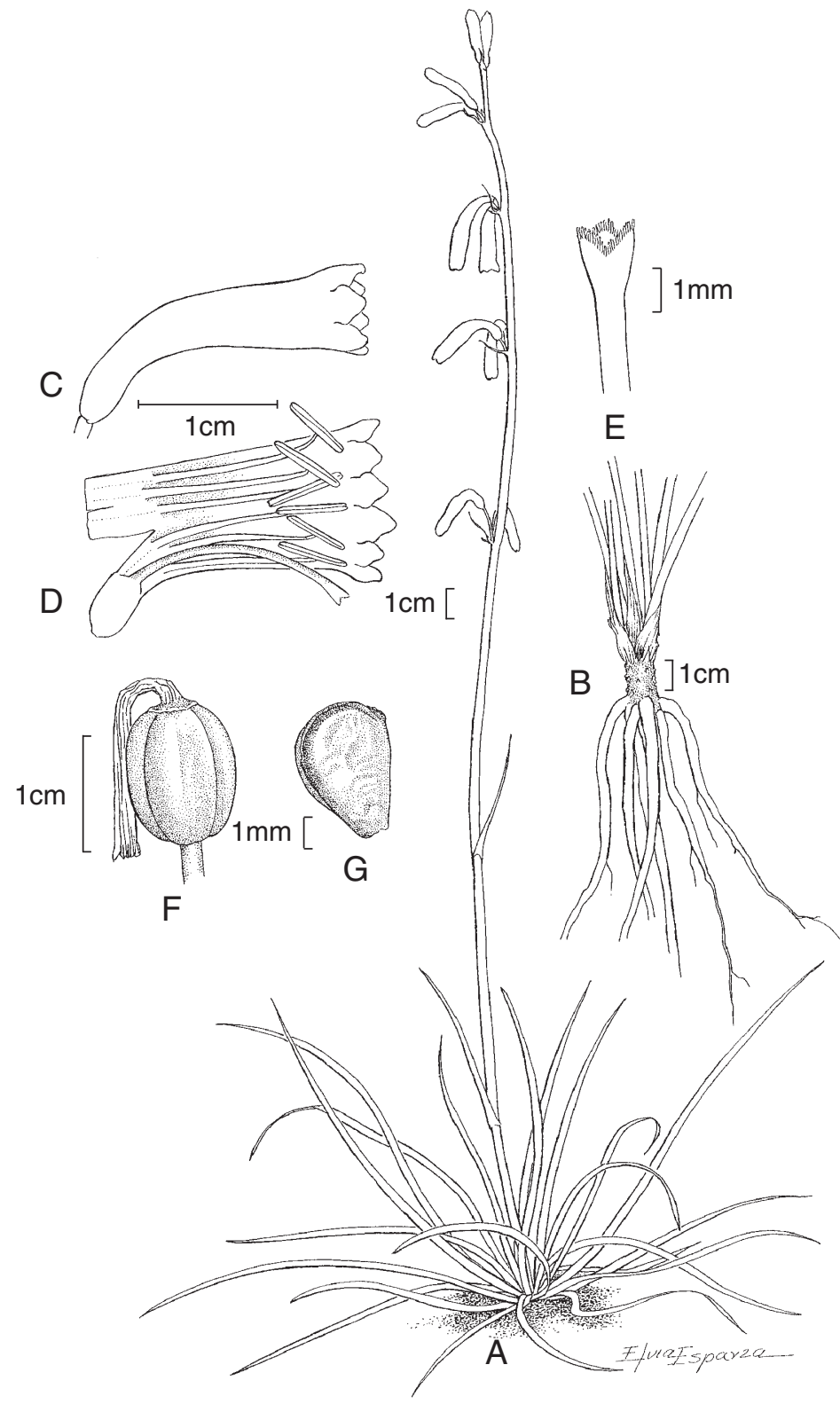

Fig. 2. Polianthes geminiflora var. pueblensis. A. hábito; B. cormo y bulbo; C. flor; D. flor disecada. E. estigma trilobado; F. fruto; G. semilla. Ilustración basada en el ejemplar $E$. Solano et al. 849 (FEZA, MEXU). 
de 4-9 cm de largo; brácteas de la base del pedúnculo reduciéndose gradualmente hacia el ápice de la inflorescencia, 11.7-15 cm de largo, 1-2 mm de ancho, lineares, ápice agudo o acuminado, margen papiloso a regularmente denticulado. Flores 1.5$2.1 \mathrm{~cm}$ de largo, 1.5-3 mm de ancho en la base de los lóbulos, geminadas, tubulares, pediceladas, colgantes en antesis, anaranjadas; pedicelos 4-13 $\mathrm{mm}$; ovario 3-6 $\mathrm{mm}$ de largo, 1-3.5 mm de diámetro; tubo 1.3-2.1 cm de largo; lóbulos 2-2.5 mm de largo, 1$2 \mathrm{~mm}$ de ancho, ovados, erectos, ápice cuculado, obtuso, con un mechón de tricomas cortos de color blanco; estambres inclusos, filamentos 1-1.7 cm de largo, insertos en la base del tubo del perigonio, 3-5 mm por arriba del ápice del ovario, porción libre de los filamentos 7-12 mm; anteras 5-6 mm de largo, lineares, inclusas, amarillas; estilo 1-1.6 cm de largo, filiforme, a veces tan largo como el tubo del perigonio; estigma trilobado, lóbulos de aproximadamente $1 \mathrm{~mm}$ de largo, aplanados, rectos, papilosos. Frutos capsulares, 1.2-1.7 cm de largo, 0.9-1.1 cm de ancho, globosos, con los restos del perigonio persistentes. Semillas 3-5 $\mathrm{mm}$ de largo, 2-3 $\mathrm{mm}$ de ancho, deltoides, planas, negras, brillantes u opacas.

Tipo: México, Puebla: municipio de Puebla, 4 km al N de la presa Manuel Ávila Camacho (Valsequillo), 2260 m, matorral de Juniperus, 12 julio 1996, E. Solano et al. 849 (holotipo: MEXU, isotipos: CHAPA, ENCB, FEZA, HUAP, HUMO, IEB, US).

Paratipos: Puebla, municipio de Amozoc, Chachapa, cerro de la derecha, M. Rodríguez 342 (HUAP); municipio de Cuauhtinchán, cerro Xonaca al norte de Cuauhtinchán, Olivas-Lazcano 895 (HUAP); carretera 528, $3 \mathrm{~km}$ antes de llegar a Cuauhtinchán, ejido San Pedro Alpatlahua, E. Solano y Ma. del C. López 853 (FEZA, MEXU); municipio de Puebla, Africam Safari en el valle de Valsequillo, M. Rodríguez 895 (HUAP); sobre la carretera a Africam Safari, enfrente del Zoológico, E. Solano et al. 794 (FEZA, MEXU); alrededores de la colonia Patria Nueva, E. Solano y C. Correa 847 (FEZA, MEXU); $2 \mathrm{~km}$ del entronque a Africam Safari, E. Solano y C. Correa 848 (FEZA, MEXU); carretera Puebla-Azomiatla, aproximadamente a $2 \mathrm{~km}$ viniendo de Azomiatla, E. Solano y C. Correa 852 (FEZA, MEXU); municipio de Tetela de Ocampo, $5 \mathrm{~km}$ al oriente de San Baltazar Tetela, $E$. Solano et al. 850 (FEZA, MEXU); entre la Libertad y la Paz Tlaxcolpa, carretera a Azomiatla, E. Solano y C. Correa 851 (FEZA, MEXU). México, sin localidad, ni fecha de recolecta, C. Ehrenberg 259 (MEXU).

Fenología: Florece de junio a agosto y fructifica de septiembre a noviembre. 
Hábitat y distribución. Se le encuentra en altitudes de 2160 a 2220 m, en áreas abiertas de bosque de Quercus, matorral xerófilo, bosque de Juniperus, pastizal y orilla de campos de cultivo de maíz, en suelos calcáreos, de color gris o negro, con textura arenosa, arcillosa o migajón-arcillosa, en poblaciones muy localizadas con pocos individuos. Se ha registrado de los municipios de: Amozoc, Cuauhtinchán, Guadalupe Victoria, Puebla y Tetela de Ocampo, en el estado de Puebla.

Se le conoce comúnmente como arete, aretito y coral. En Cuauhtinchán, según la información de la etiqueta del ejemplar Olivas-Lazcano 895, se emplea como cicatrizante, aunque no se indica ni la forma de uso, ni la parte usada. En su área de distribución las plantas sirven de alimento al ganado bovino y caprino.

Polianthes geminiflora pertenece al subgénero Bravoa; es la especie con distribución geográfica más amplia del género. Se encuentra desde Durango y Nayarit en el noroeste, y el norte de Veracruz en sus límites con Hidalgo en el noreste, hasta Guerrero y Puebla. Crece en tipos de vegetación muy diversos, como bosque templado, matorral xerófilo, pastizal y selva mediana subcaducifolia. Debido a esta amplitud geográfica y ecológica presenta una gran variación morfológica.

Con base en el tipo de margen de la hoja, McVaugh (1989a) reconoció para la región de Nueva Galicia tres variedades: Polianthes geminiflora var. geminiflora, $P$. geminiflora var. clivicola y $P$. geminiflora var. graminifolia. Polianthes geminiflora var. geminiflora se encuentra en las montañas de Durango, Nayarit, algunas áreas de Jalisco y Michoacán, Querétaro, Estado de México, Hidalgo, Tlaxcala, norte de Puebla, Morelos, Guerrero y Veracruz, presenta hojas de 0.5$1.5 \mathrm{~cm}$ de ancho y margen papiloso o verrucoso (a veces se alternan porciones lisas con papilosas). Polianthes geminiflora var. clivicola se distribuye en las zonas montañosas más húmedas del sur de Jalisco y norte de Michoacán, son plantas más grandes que la variedad típica, con inflorescencias más largas y hojas más anchas (generalmente de 0.4-3.7 cm), margen liso, translúcido, hialino o escarioso. Polianthes geminiflora var. graminifolia es considerada como una especie distinta, tal como fue propuesta originalmente por Rose (1903) y apoyada por los estudios fenéticos y cladísticos de Solano (2000). Polianthes graminifolia se localiza en el sur de Zacatecas, centro-norte de Jalisco y sur de Aguascalientes, las hojas miden de 0.5-0.8 cm de ancho, margen ciliado, así como el envés y la base del pedúnculo floral pubescentes. 
En el centro de Puebla, límites con el sur de Tlaxcala, en ambientes más xerofíticos y áreas abiertas de matorral, se localizan las poblaciones de Polianthes geminiflora var. pueblensis, con hojas de 0.1-0.2 cm de ancho, papilosas en el envés, margen papiloso a regularmente denticulado y frutos ligeramente mayores que en las variedades anteriores. El ejemplar de herbario C. Ehrenberg 259 (3 exsiccata MEXU), sin localidad, ni fecha (al parecer recolectado en la tercera década del siglo XIX), está determinado como Bravoa geminiflora var. angustifolia K1.; sin embargo, esta combinación no fue publicada.

De acuerdo con Solano (2000), se reconocen dos de las tres variedades propuestas por McVaugh (1989a) y se consideran a las poblaciones del centro de Puebla como pertenecientes a una variedad nueva. En el Cuadro 2 se muestran las diferencias morfológicas y anatómicas entre las tres variedades de Polianthes geminiflora y Polianthes graminifolia. Polianthes geminiflora var. pueblensis, se distingue anatómicamente por carecer de taninos en las células del mesofilo, así como de rafidios en las células subepidérmicas del pedúnculo y presentar pared secundaria delgada entre la región central del pedúnculo y la epidermis. Estos taxa se separan de acuerdo con la siguiente clave.

1 Márgenes de las hojas ciliados, envés y base del pedúnculo floral pubescente P. graminifolia

1 Márgenes de las hojas lisos, papilosos a regularmente denticulados, envés papiloso, base del pedúnculo floral glabro.

2 Hojas 0.1-0.2 cm de ancho P. geminiflora var. pueblensis

2 Hojas (0.4-)1.5-2.5(-3.7) cm de ancho.

3 Margen de la hoja translúcido, hialino o escarioso, liso, angosto, revoluto .... P. geminiflora var. clivicola

3 Margen de la hoja papiloso o verrucoso, a veces alternándose porciones lisas con papilosas P. geminiflora var. geminiflora

\section{AGRADECIMIENTOS}

Agradecemos a Fernando Chiang y Raquel Galván la revisión crítica del manuscrito; el primero elaboró las diagnosis en latín. Teresa Terrazas asesoró el trabajo anatómico, Elvia Esparza realizó las ilustraciones y los curadores de los herbarios CHAP, FEZA, HUAP y MEXU nos permitieron la consulta de los ejemplares. 
Cuadro 2. Diferencias morfológicas y anatómicas entre las variedades de Polianthes geminiflora (Lex.) Rose y Polianthes graminifolia Rose.

\begin{tabular}{|c|c|c|c|c|}
\hline Carácter & $\begin{array}{l}\text { P. geminiglora } \\
\text { var. pueblensis }\end{array}$ & $\begin{array}{l}\text { P. geminiflora } \\
\text { var. clivicola }\end{array}$ & $\begin{array}{c}\text { P. geminiflora } \\
\text { var. geminiflora }\end{array}$ & $\begin{array}{c}P . \\
\text { graminifolia }\end{array}$ \\
\hline Número de hojas & $3-6$ & $2-4(-8)$ & $2-4$ & $3-4$ \\
\hline Tamaño de hojas & $\begin{array}{l}14-17 \times 0.1- \\
0.2 \mathrm{~cm}\end{array}$ & $\begin{array}{l}12-35(-43) \times 0.4- \\
1.5(3.7) \mathrm{cm}\end{array}$ & $\begin{array}{l}13-25(-36) \times \\
0.5-1.5 \mathrm{~cm}\end{array}$ & $\begin{array}{l}15-22 \times 0.1- \\
0.2 \mathrm{~cm}\end{array}$ \\
\hline Forma de la hoja & linear & $\begin{array}{l}\text { linear a } \\
\text { lanceolada }\end{array}$ & $\begin{array}{l}\text { linear a } \\
\text { lanceolada }\end{array}$ & linear \\
\hline Margen & $\begin{array}{l}\text { papiloso a } \\
\text { regularmente } \\
\text { denticulado }\end{array}$ & $\begin{array}{l}\text { liso, translúcido, } \\
\text { hialino o } \\
\text { escarioso }\end{array}$ & $\begin{array}{l}\text { papiloso o ve- } \\
\text { rrucoso, a veces } \\
\text { alternándose } \\
\text { porciones lisas } \\
\text { con papilosas }\end{array}$ & ciliado \\
\hline $\begin{array}{l}\text { Tamaño de la } \\
\text { inflorescencia }\end{array}$ & $35-65 \mathrm{~cm}$ & $\begin{array}{l}(21-) 45-100(-154) \\
\mathrm{cm}\end{array}$ & $\begin{array}{l}(23-) 40-90(-125) \\
\mathrm{cm}\end{array}$ & $\begin{array}{l}(20-) 50-85 \\
\mathrm{~cm}\end{array}$ \\
\hline Fruto & $\begin{array}{l}1.2-1.7 \times 0.9- \\
1.1 \mathrm{~cm}\end{array}$ & $0.6-1 \times 0.7-0.9 \mathrm{~cm}$ & $\begin{array}{l}0.7-1(-1.7) \mathrm{x} \\
0.5-1 \mathrm{~cm}\end{array}$ & $1 \times 1 \mathrm{~cm}$ \\
\hline Largo de semilla & $3-5 \mathrm{~mm}$ & $1.9-2.2 \mathrm{~mm}$ & $2.5-3 \mathrm{~mm}$ & $3.5-4 \mathrm{~mm}$ \\
\hline $\begin{array}{l}\text { Taninos en las cé- } \\
\text { lulas del mesófilo }\end{array}$ & ausentes & presentes & presentes & presentes \\
\hline $\begin{array}{l}\text { Cuerpos de sílice } \\
\text { en el parénquima } \\
\text { de la región cen- } \\
\text { tral del cormo* }\end{array}$ & ausentes & ausentes & presentes & ausentes \\
\hline $\begin{array}{l}\text { Epidermis del } \\
\text { pedúnculo* }\end{array}$ & lisa & papilosa & lisa & papilosa \\
\hline $\begin{array}{l}\text { Rafidios en células } \\
\text { subepidérmicas } \\
\text { del pedúnculo* }\end{array}$ & ausentes & presentes & presentes & presentes \\
\hline $\begin{array}{l}\text { Taninos en células } \\
\text { subepidérmicas } \\
\text { del pedúnculo* }\end{array}$ & ausentes & ausentes & presentes & ausentes \\
\hline $\begin{array}{l}\text { Pared secundaria } \\
\text { entre la región } \\
\text { central del } \\
\text { pedúnculo y la } \\
\text { epidermis* }\end{array}$ & delgada & gruesa & gruesa & gruesa \\
\hline
\end{tabular}

* Caracteres tomados de González (1998). 


\section{LITERATURA CITADA}

Bogler, D. J. y B. B. Simpson. 1996. Phylogeny of Agavaceae based on ITS RDNA sequence variation. Amer. J. Bot. 83: 1225-1235.

Eguiarte, L. E., V. Souza y A. Silva-Montellano. 2000. Evolución de la familia Agavaceae: filogenia, biología reproductiva y genética de poblaciones. Bol. Soc. Bot. Méx. 66: $131-150$.

González, A. 1998. Descripción morfológica y anatómica del tallo de Polianthes L. (Agavaceae). Tesis de licenciatura. Facultad de Estudios Superiores Zaragoza, Universidad Nacional Autónoma de México. México, D.F. 43 pp.

McVaugh, R. 1989a. Polianthes L. In: Anderson, W. R. (ed.). Flora Novo-Galiciana 15: 247260.

McVaugh, R. 1989b. Prochnyanthes S. Wats. In: Anderson, W. R. (ed.). Flora NovoGaliciana 15: 260-263.

Rose, J. N. 1903. Studies of Mexican and Central American plants. Revision of Polianthes with new species. Contr. U.S. Natl. Herb. 8: 8-13.

Solano, E. 2000. Sistemática del género Polianthes L. (Agavaceae). Tesis doctoral. Facultad de Ciencias, Universidad Nacional Autónoma de México. México, D.F. 291 pp.

Solano, E. y A. García-Mendoza. 1998. Nueva especie de Polianthes (Agavaceae) del estado de Oaxaca, México. Sida Contributions to Botany 18(2): 473-477.

Verhoek-W., S. E. 1975. A study of the tribe Poliantheae (including Manfreda) and revisions of Manfreda and Prochnyanthes (Agavaceae). Tesis doctoral. Cornell University, Ithaca, Nueva York. 405 pp. 